\title{
Maximal classes for families of lower and upper semicontinuous functions with a closed graph
}

\author{
Jolanta Kosman ${ }^{1}$
}

Received: 12 February 2016 / Accepted: 23 August 2016/ Published online: 22 September 2016

(C) The Author(s) 2016. This article is published with open access at Springerlink.com

\begin{abstract}
In this paper we characterize the following maximal classes for families of lower and upper semicontinuous functions with a closed graph: the maximal additive class, the maximal multiplicative class and the maximal classes with respect to maximum and minimum.
\end{abstract}

Keywords Functions with a closed graph - Lower semicontinuous functions - Upper semicontinuous functions $\cdot$ Sum of functions

Mathematics Subject Classification Primary 26A15; Secondary 54C08

\section{Introduction}

The letters $\mathbb{R}, \mathbb{Q}$ and $\mathbb{N}$ denote the real line, the set of rationals and the set of positive integers, respectively. The family of all functions from a set $X$ into $Y$ is denoted by $Y^{X}$. For each set $A \subset X$ its characteristic function is denoted by $\chi_{A}$. In particular, $\chi \emptyset_{\emptyset}$ stands for the zero constant function.

Let $X$ be a topological space. The symbol $X^{d}$ denotes the set of all accumulation points of $X$. For each set $A \subset X$ the symbols int $A$ and $\operatorname{cl} A$ denote the interior and the closure of $A$, respectively. The spaces $\mathbb{R}$ and $X \times \mathbb{R}$ are considered with their standard topologies. We say that a function $f: X \rightarrow \mathbb{R}$ has a closed graph, if the graph of $f$, i.e., the set $\{(x, f(x)): x \in X\}$ is a closed subset of the product $X \times \mathbb{R}$. We say that a function $f: X \rightarrow \mathbb{R}$ is lower (upper) semicontinuous at a point $x \in X$, if for each $\varepsilon>0$ there is an open neighborhood $U$ of $x$ such that $f(z)>f(x)-\varepsilon(f(z)<f(x)+\varepsilon$, respectively) for each $z \in U$. If $f: X \rightarrow \mathbb{R}$ is lower (upper) semicontinuous at each point $x \in X$, then we say that the function $f$ is lower (upper, respectively) semicontinuous. Let $\mathscr{C}$ onst $(X), \mathcal{C}(X)$,

Jolanta Kosman

jola.kosman@wp.pl

1 Institute of Mathematics, Kazimirz Wielki University, pl. Weyssenhoffa 11, 85-072 Bydgoszcz, Poland 
$\mathcal{U}(X), \operatorname{lsc}(X), \operatorname{usc}(X)$ denote the class of all real-valued functions on $X$ that are constant, continuous, have a closed graph, are lower and upper semicontinuous, respectively. Obviously $\mathcal{C}(X) \subset \mathcal{U}(X)$ (see also e.g. [5]) and $C(X)=l s c(X) \cap u s c(X)$. For $\mathcal{F}(X)$ and $\mathcal{G}(X)$ nonempty subsets of $\mathbb{R}^{X}$ the symbol $\mathcal{F} \mathcal{G}(X)$ denotes the class $\mathcal{F}(X) \cap \mathcal{G}(X)$. Further denote by $\mathcal{F}^{+}(X)$ the family of all nonnegative functions from $\mathcal{F}(X)$. Let $f \in \mathbb{R}^{X}$. The symbol $G(f)$ denotes the graph of $f$ and the symbols $C(f)$ and $D(f)$ denote the sets of points of continuity and discontinuity of $f$, respectively. For each $y \in \mathbb{R}$ let $[f=y]=\{x \in X: f(x)=y\}$. Similarly we define the symbols $[f>y],[f<y]$.

If $\mathcal{F} \subset \mathbb{R}^{X}$ is a family of functions, denote by

$$
\begin{aligned}
& \mathcal{F}+\mathcal{F} \stackrel{\text { df }}{=}\left\{f \in \mathbb{R}^{X}: f=g+h \text { for some } g, h \in \mathcal{F}\right\}, \\
& \mathcal{M}_{a}(\mathcal{F}) \stackrel{\text { df }}{=}\left\{f \in \mathbb{R}^{X}:\left(\forall_{g \in \mathcal{F}}\right) f+g \in \mathcal{F}\right\}, \\
& \mathcal{M}_{m}(\mathcal{F}) \stackrel{\text { df }}{=}\left\{f \in \mathbb{R}^{X}:\left(\forall_{g \in \mathcal{F}}\right) f \cdot g \in \mathcal{F}\right\}, \\
& \mathcal{M}_{\text {max }}(\mathcal{F}) \stackrel{\text { df }}{=}\left\{f \in \mathbb{R}^{X}:\left(\forall_{g \in \mathcal{F}}\right) \max (f, g) \in \mathcal{F}\right\}, \\
& \mathcal{M}_{\text {min }}(\mathcal{F}) \stackrel{\text { df }}{=}\left\{f \in \mathbb{R}^{X}:\left(\forall_{g \in \mathcal{F}}\right) \min (f, g) \in \mathcal{F}\right\} .
\end{aligned}
$$

The above classes $\mathcal{M}_{a}(\mathcal{F}), \mathcal{M}_{m}(\mathcal{F}), \mathcal{M}_{\max }(\mathcal{F})$ and $\mathcal{M}_{\min }(\mathcal{F})$ are called the maximal additive class for $\mathcal{F}$, the maximal multiplicative class for $\mathcal{F}$, the maximal class with respect to maximum and minimum for $\mathcal{F}$, respectively.

In 1987 Menkyna [7] characterized the maximal additive and multiplicative classes for the family of functions with a closed graph. He proved that $\mathcal{M}_{a}(\mathcal{U}(X))=\mathcal{C}(X)$ for a topological space $X$ [7, Theorem 1] and $\mathcal{M}_{m}(\mathcal{U}(X))=\{f \in \mathcal{C}(X):[f=0]$ is an open set $\}$ for a locally compact normal topological space $X$ [7, Theorem 2]. Let $\mathcal{Q}(X)$ denote the family of all quasi-continuous functions from a topological space $X$ to $\mathbb{R}$. Recall that $f \in \mathcal{Q}(X)$ if and only if for each $x \in X, \varepsilon>0$ and for each neighbourhood $U$ of $x$ there is a nonempty open set $V \subset U$ such that $|f(x)-f(y)|<\varepsilon$ for each $y \in V$. In 2008 Sieg [8] considered real functions defined on $\mathbb{R}$ and showed that $\mathcal{M}_{a}(\mathcal{Q U}(\mathbb{R}))=\mathcal{C}(\mathbb{R}), \mathcal{M}_{m}(\mathcal{Q U}(\mathbb{R}))=\{f \in$ $\mathcal{C}(\mathbb{R}): f=\chi \emptyset$ or $f(x) \neq 0$ for all $x \in \mathbb{R}\}$ and $\mathcal{M}_{\max }(\mathcal{Q U}(\mathbb{R}))=\mathcal{M}_{\min }(\mathcal{Q U}(\mathbb{R}))=\emptyset$. In 2014 Szczuka (see $[9,10]$ ) characterized the following maximal classes for lower and upper semicontinuous strong Świątkowski functions and lower and upper semicontinuous extra strong Świątkowski functions: the maximal additive class, the maximal multiplicative class and the maximal classes with respect to maximum. She proved, among others, that if $\mathcal{F}$ denotes the family of lower semicontinuous strong Świątkowski real functions defined on $\mathbb{R}$, then $\mathcal{M}_{a}(\mathcal{F})=$ Const $\left[9\right.$, Theorems 3.1], $\mathcal{M}_{m}(\mathcal{F})=\mathscr{C}_{\text {onst }}{ }^{+}[9$, Theorem 3.2] and $\mathcal{M}_{\max }(\mathcal{F})=\mathscr{C}$ onst $[9$, Theorem 3.3].

In this paper we deal with the families of lower and upper semicontinuous functions with a closed graph. We obtain the following results:

- $\mathcal{M}_{a}(\mathcal{U} l s c(X))=\mathcal{U} l s c(X)$, where $X$ is a topological space (Theorem 2.5),

- $\mathcal{M}_{a}(\mathcal{U} u s c(X))=\mathcal{U} u s c(X)$, where $X$ is a topological space (Theorem 3.3),

- $\mathcal{M}_{m}(\mathcal{U} l s c(X))=\{f \in \mathcal{C}(X):[f=0]$ is an open set and $f(x) \geq 0$ for all $x \in X\}=$ $\mathcal{M}_{m}(\mathcal{U} u s c(X))$, where $X$ is a perfectly normal topological space such that $X=X^{d}$ (Theorems 2.7, 3.4),

- $\mathcal{M}_{\max }(\mathcal{U} l s c(X))=\mathcal{U} l s c(X)$, where $X$ is a topological space (Theorem 2.10),

- $\mathcal{M}_{\min }(\mathcal{U} u s c(X))=\mathcal{U} u s c(X)$, where $X$ is a topological space (Theorem 3.5),

- $\mathcal{M}_{\min }(\mathcal{U} l s c(X))=\mathcal{M}_{\max }(\mathcal{U} u s c(X))=\emptyset$, where $X$ is a perfectly normal topological space such that $X^{d} \neq \varnothing$ (Corollary 2.15, Theorems 3.6). 


\section{Lower semicontinuous functions with a closed graph}

We start with a following proposition.

Proposition 2.1 Let $X$ be a topological space. A function $f: X \rightarrow \mathbb{R}$ has the closed graph if and only if for each $x \in X$ and for each $m \in \mathbb{N}$ there is a neighbourhood $V$ of $x$ such that $f(z) \in(-\infty,-m) \cup(f(x)-1 / m, f(x)+1 / m) \cup(m, \infty)$ for each $z \in V$.

Proof The implication $(\Leftarrow)$ we can find in [2] (see p. 118, lines 11-14). The implication $(\Rightarrow)$ immediately follows from [6] or [1, Proposition 1]: if $f \in \mathcal{U}(X)$, then for each $x \in X$ and each neighborhood $U$ of $f(x)$ such that $Y \backslash U$ is compact there is an neighborhood $V$ of $x$ such that $f(V) \subset U$. Now, it is sufficient to take $U=(-\infty,-m) \cup(f(x)-1 / m, f(x)+$ $1 / m) \cup(m, \infty)$. Observe that, the equivalence of this proposition also immediately follows from [1, Proposition 2].

From above and the definitions of the class $l s c$ we obtain:

Lemma 2.2 Let $X$ be a topological space. A function $f: X \rightarrow \mathbb{R}$ is lower semicontinuous function with a closed graph if and only if for each $x \in X$ and for each $m \in \mathbb{N}$ there is a neighbourhood $V$ of $x$ such that $f(z) \in(f(x)-1 / m, f(x)+1 / m) \cup(m, \infty)$ for each $z \in V$.

Proof First, assume that for each $x \in X$ and for each $m \in \mathbb{N}$ there is a neighbourhood $V$ of $x$ such that $f(z) \in(f(x)-1 / m, f(x)+1 / m) \cup(m, \infty)$ for each $z \in V$. Then, by Proposition 2.1, $f \in \mathcal{U}(X)$. Now, we will show that $f \in l \operatorname{sc}(X)$. Let $x \in X$ and $\varepsilon>0$. We choose $m \in \mathbb{N}$ such that $m \geq \max \left\{\frac{1}{\varepsilon}, f(x)-\varepsilon\right\}$. There is a neighbourhood $V$ of $x$ such that $f(z) \in(f(x)-1 / m, f(x)+1 / m) \cup(m, \infty) \subset(f(x)-\varepsilon, \infty)$ for each $z \in V$ and consequently $f \in l s c(X)$.

Now, let $f \in \mathcal{U} l s c(X)$. Fix $x \in X$ and $m \in \mathbb{N}$. Since $f \in l s c(X)$, there is a neighbourhood $V_{1}$ of $x$ such that $f(z) \in(f(x)-1 / m, \infty)$ for each $z \in V_{1}$. We consider two cases.

First, assume that $f(x) \geq 0$. Since $f \in \mathcal{U}(X)$, there is a neighbourhood $V_{2}$ of $x$ such that $f(z) \in(-\infty,-m) \cup(f(x)-1 / m, f(x)+1 / m) \cup(m, \infty)$ for each $z \in V_{2}$ (see Proposition 2.1). Let $V=V_{1} \cap V_{2}$ and let $z \in V$. Then $f(z) \in(f(x)-1 / m, f(x)+1 / m) \cup$ $(m, \infty)$.

Now, assume that $f(x)<0$. We choose $k \in \mathbb{N}$ such that $k \geq \max \left\{m,-f(x)+\frac{1}{m}\right\}$. Since $f \in \mathcal{U}(X)$, there is a neighbourhood $V_{2}$ of $x$ such that $f(z) \in(-\infty,-k) \cup(f(x)-$ $1 / k, f(x)+1 / k) \cup(k, \infty)$ for each $z \in V_{2}$. Let $V=V_{1} \cap V_{2}$ and let $z \in V$. Since $k \geq m$, $-k \leq f(x)-\frac{1}{m}$ and $\frac{1}{k} \leq \frac{1}{m}$, we have $f(z) \in((f(x)-1 / k, f(x)+1 / k) \cup(k, \infty)) \subset$ $((f(x)-1 / m, f(x)+1 / m) \cup(m, \infty))$. This completes the proof.

The next lemma follows from Proposition 2.1 and Lemma 2.2.

Lemma 2.3 Let $X$ be a topological space. Then $\mathcal{U}^{+}(X) \subset \mathcal{U} l s c(X)$.

Now, we will characterize the class of the sums of lower semicontinuous functions with a closed graph.

Lemma 2.4 Let $X$ be a topological space. Then $\mathcal{U l s c}(X)+\mathcal{U l s c}(X)=\mathcal{U} l s c(X)$.

Proof Let $f, g \in \mathcal{U} l s c(X)$. Fix $x \in X$ and $m \in \mathbb{N}$. Let $k \in \mathbb{N}$ be such that $1 / k<1 /(2 m+$ $|f(x)|+|g(x)|)$. By Lemma 2.2, there exists a neighbourhood $V$ of $x$ such that $f(z) \in$ 
$(f(x)-1 / k, f(x)+1 / k) \cup(k, \infty)$ and $g(z) \in(g(x)-1 / k, g(x)+1 / k) \cup(k, \infty)$ for each $z \in V$. Let $z \in V$. We consider four cases.

If $f(z)>k$ and $g(z)>k$, then evidently $(f+g)(z)>m$.

If $f(z)>k$ and $g(z) \in(g(x)-1 / k, g(x)+1 / k)$, then

$$
(f+g)(z)>k+g(x)-1 / k>2 m+|f(x)|+|g(x)|+g(x)-1 / k>m .
$$

Similarly, $g(z)>k$ and $f(z) \in(f(x)-1 / k, f(x)+1 / k)$, implies $(f+g)(z)>m$.

Now, let $f(z) \in(f(x)-1 / k, f(x)+1 / k)$ and $g(z) \in(g(x)-1 / k, g(x)+1 / k)$. Then, we have

$$
|(f+g)(z)-(f+g)(x)| \leq|f(z)-f(x)|+|g(z)-g(x)|<2 / k<1 / m .
$$

It follows that $f+g \in \mathcal{U} l s c(X)$.

Theorem 2.5 Let $X$ be a topological space. Then $\mathcal{M}_{a}(\mathcal{U l s c}(X))=\mathcal{U} l s c(X)$.

Proof Since $\chi_{\emptyset} \in \mathcal{U} l s c(X)$, we conclude that $\mathcal{M}_{a}(\mathcal{U l s c}(X)) \subset \mathcal{U l s c}(X)$. The inclusion $\mathcal{U} l s c(X) \subset \mathcal{M}_{a}(\mathcal{U} l s c(X))$ follows from Lemma 2.4 .

Now, recall the following lemma [7, Lemma 2], which will be applied in this paper.

Proposition 2.6 Let $X$ be a topological space and let $f \in \mathcal{C}(X)$. Then the function $g: X \rightarrow$ $\mathbb{R}$ defined by the formula

$$
g(x)= \begin{cases}\frac{1}{f(x)}, & \text { if } \mathrm{x} \in[\mathrm{f} \neq 0] \\ 0, & \text { if } \mathrm{x} \in[\mathrm{f}=0] .\end{cases}
$$

has the closed graph.

Theorem 2.7 Let $X$ be a normal topological space such that each singleton is $G_{\delta}$-set. Then

$$
\mathcal{M}_{m}(\mathcal{U l s c}(X))=\left\{f \in \mathcal{C}(X):[f=0] \text { is an open set and }[f<0]^{d}=\emptyset\right\} .
$$

Proof We will prove this theorem in four parts. First, we will show that $\mathcal{M}_{m}(\mathcal{U l s c}(X)) \subset$ $\mathcal{C}(X)$. Let $f \in \mathcal{M}_{m}(\mathcal{U} l s c(X))$. Since $\chi_{\mathbb{R}},-\chi_{\mathbb{R}} \in \mathcal{U} l s c(X)$, we have $f \in l s c(X)$ and $-f \in l s c(X)$. Consequently $f \in l \operatorname{sc}(X) \cap \operatorname{usc}(X)=\mathcal{C}(X)$.

Now, we assume that the function $f \in \mathcal{C}(X)$ and the set $[f=0]$ is not open. We will show that $f \notin \mathcal{M}_{m}(\mathcal{U} l s c(X))$ (The proof of this part is similar to the second part of the proof of [7, Theorem 2]). Define the function $g: X \rightarrow \mathbb{R}$ by the formula

$$
g(x)= \begin{cases}\frac{1}{|f(x)|}, & \text { if } \mathrm{x} \in[\mathrm{f} \neq 0], \\ 0, & \text { if } \mathrm{x} \in[\mathrm{f}=0] .\end{cases}
$$

By Proposition 2.6 the function $g$ has the closed graph. Moreover $g$ is non-negative function and consequently, by Lemma 2.3, $g \in \mathcal{U} l s c(X)$. Now, we will show that $f \cdot g \notin \mathcal{U} l s c(X)$.

Since the set $[f=0]$ is not open, there is $x_{0} \in[f=0]$ such that for each open neighbourhood $V$ of $x_{0}$ there is $x_{V} \in V \cap[f \neq 0]$. Notice that $(f \cdot g)\left(x_{V}\right) \in\{-1,1\}$ for each neighbourhood $V$ of $x_{0}$ and $(f \cdot g)\left(x_{0}\right)=0$. By Proposition 2.1, $f \cdot g \notin \mathcal{U}(X)$.

In the third part of the proof, suppose that $f \in \mathcal{C}(X)$, the set $[f=0]$ is open and $[f<0]^{d} \neq \varnothing$. We will prove that $f \notin \mathcal{M}_{m}(\mathcal{U} l s c(X))$. Let $x_{0} \in[f<0]^{d}$. Then there is a net $\left(x_{\gamma}\right)_{\gamma \in \Gamma}$ of elements of $X$ such that $x_{\gamma} \rightarrow x_{0}, x_{\gamma} \neq x_{0}$ and $f\left(x_{\gamma}\right)<0$ for every $\gamma \in \Gamma$. 
Notice that, since $f \in \mathcal{C}(X)$ and the set $[f=0]$ is open, we have $f\left(x_{0}\right)<0$. By Urysohn Lemma there is a continuous function $h: X \rightarrow[0,1]$ such that $[h=0]=\left\{x_{0}\right\}$.

Define the function $g: X \rightarrow \mathbb{R}$ by the formula

$$
g(x)= \begin{cases}\frac{1}{h(x)}, & \text { if } \mathrm{x} \neq \mathrm{x}_{0}, \\ 0, & \text { if } \mathrm{x}=\mathrm{x}_{0} .\end{cases}
$$

Observe that, by Proposition 2.6 and Lemma 2.3, $g \in \mathcal{U} l s c(X)$. Moreover $f \cdot g \notin l s c(X)$, because the net $\left((f \cdot g)\left(x_{\gamma}\right)\right)_{\gamma \in \Gamma}$ diverges to $-\infty$ (recall that $\left.f\left(x_{\gamma}\right) \rightarrow f\left(x_{0}\right)<0\right)$.

In the last part suppose that $f \in \mathcal{C}(X)$, the set $[f=0]$ is open, $[f<0]^{d}=\varnothing$ and $g \in \mathcal{U l s c}(X)$. Then, by [7, Theorem 2], $(f \cdot g) \in \mathcal{U}(X)$ (see also the third part of the proof of [7, Theorem 2]). It is enough to show that $(f \cdot g) \in l s c(X)$. Let $x_{0} \in X$. If $f\left(x_{0}\right) \leq 0$, then the function $f \cdot g$ is continuous at $x_{0}$ and consequently $f \cdot g$ is a lower semicontinuous at this point. Indeed, if $f\left(x_{0}\right)=0$, then by the assumption $[f=0]=\operatorname{int}[f=0]$, we have $x_{0} \in \operatorname{int}[f=0] \subset \operatorname{int}[f \cdot g=0]$ and if $f\left(x_{0}\right)<0$, then $x_{0}$ is a isolated point of $X$. Finally, assume that $f\left(x_{0}\right)>0$. Since $f \in \mathcal{C}(X)$, there is an open neighborhood $U$ of $x_{0}$ such that $U \subset[f>0]$. Since $g \in l s c(X), f$ is continuous and positive function on $U$, the function $f \cdot g$ is a lower semicontinuous at $x_{0}$. The proof is complete.

It is easy to see that from above for $X=\mathbb{R}$ we have the following corollary.

Corollary 2.8 $\mathcal{M}_{m}(\mathcal{U l s c}(\mathbb{R}))=\left\{f \in \mathcal{C}(\mathbb{R}): f=\chi_{\emptyset}\right.$ or $f(x)>0$ for all $\left.x \in \mathbb{R}\right\}$.

Lemma 2.9 Let $X$ be a topological space and let $f, g \in \mathcal{U l s c}(X)$. Then the real function $h=\max \{f, g\}$ defined on $X$ is a lower semicontinuous function with a closed graph.

Proof Let $f, g \in \mathcal{U} l s c(X)$. We will use Lemma 2.2. Fix $x \in X$ and $m \in \mathbb{N}$. Then there exists a neighbourhood $V$ of $x$ such that $f(z) \in(f(x)-1 / m, f(x)+1 / m) \cup(m, \infty)$ and $g(z) \in(g(x)-1 / m, g(x)+1 / m) \cup(m, \infty)$ for each $z \in V$. We assume that $f(x) \geq g(x)$ (The case $f(x)<g(x)$ is analogous). Then $h(x)=f(x)$ and it is easy to see that $h(z) \in$ $(h(x)-1 / m, h(x)+1 / m) \cup(m, \infty)$ for each $z \in V$. So, $h \in \mathcal{U} l s c(X)$.

Theorem 2.10 Let $X$ be a topological space. Then $\mathcal{M}_{\max }(\mathcal{U l s c}(X))=\mathcal{U} l s c(X)$.

Proof The inclusion $\mathcal{U} l s c(X) \subset \mathcal{M}_{\max }(\mathcal{U} l s c(X))$ follows from Lemma 2.9. So, we will only prove that $\mathcal{M}_{\max }(\mathcal{U} l s c(X)) \subset \mathcal{U} l s c(X)$. Let $f: X \rightarrow \mathbb{R}$ be a function such that $f \notin \mathcal{U l} l s c(X)$. We choose $x_{0} \in X$ and $m \in \mathbb{N}$, such that $m \geq f\left(x_{0}\right)+\frac{1}{m}$ and for each open neighborhood $V$ of $x_{0}$ there is $x \in V$ such that $f(x) \leq m$ and $f(x) \notin\left(f\left(x_{0}\right)-\frac{1}{m}, f\left(x_{0}\right)+\frac{1}{m}\right)$. We will show that $f \notin \mathcal{M}_{\max }(\mathcal{U} l s c(X))$. Let $c=f\left(x_{0}\right)-\frac{1}{m}$. Define the function $g: X \rightarrow \mathbb{R}$ by $g \stackrel{\text { df }}{=} c$. Clearly $g \in \mathcal{U} l s c(X)$. Denote $h=\max \{f, g\}$. We will prove that $h \notin \mathcal{U} l s c(X)$. Notice that $h\left(x_{0}\right)=f\left(x_{0}\right)$. Observe that, for each open neighborhood $V$ of $x_{0}$ there is $x_{V} \in V$ such that $f\left(x_{V}\right) \in(-\infty, c] \cup\left[f\left(x_{0}\right)+\frac{1}{m}, m\right]$ and consequently $h\left(x_{V}\right) \in\left\{h\left(x_{0}\right)-\frac{1}{m}\right\} \cup\left[h\left(x_{0}\right)+\frac{1}{m}, m\right]$. By Proposition 2.1, $h \notin \mathcal{U}(X)$. This completes the proof.

Theorem 2.11 Let $X$ be a topological space such that $\mathcal{U}(X) \neq \mathcal{C}(X)$. Then $\mathcal{M}_{\min }(\mathcal{U l s c}(X))=$ $\emptyset$.

Proof Let $f \in \mathbb{R}^{X}$. We will show that there is a function $g \in \mathcal{U l s c}(X)$ such that the function $h=\min \{f, g\} \notin \mathcal{U} l s c(X)$.

Let $g_{1}: X \rightarrow \mathbb{R}$ be a function with a closed graph and let $x_{0} \in D\left(g_{1}\right)$. Put $g_{2}=\left|g_{1}\right|$. Then $g_{2} \in \mathcal{U} l s c$ and there is a net $\left(x_{\gamma}\right)_{\gamma \in \Gamma}$ of elements of $X$ which converges to the point $x_{0}$ and a net $\left(g_{2}\left(x_{\gamma}\right)\right)_{\gamma \in \Gamma}$ diverges to $\infty$. We consider two cases. 
If $x_{0} \in C(f)$, we define the function $g: X \rightarrow \mathbb{R}$ by $g(x) \stackrel{\text { df }}{=} g_{2}(x)-g_{2}\left(x_{0}\right)+f\left(x_{0}\right)-1$. It is easy to see that $g \in \mathcal{U l s c}(X)$. Let $h=\min \{f, g\}$. Then $h\left(x_{0}\right)=g\left(x_{0}\right)=f\left(x_{0}\right)-1$ and there is $\gamma_{0} \in \Gamma$ such that $h\left(x_{\gamma}\right)=f\left(x_{\gamma}\right)$ for each $\gamma>\gamma_{0}$. Consequently $\left(x_{0}, f\left(x_{0}\right)\right) \in$ $\operatorname{cl} G(h) \backslash G(h)$ and $h \notin \mathcal{U}(X)$.

Now, let $x_{0} \in D(f)$. There is $\varepsilon>0$ such that for each neighbourhood $V$ of $x_{0}$ there is $z \in V$ such that $f(z) \notin\left(f\left(x_{0}\right)-\varepsilon, f\left(x_{0}\right)+\varepsilon\right)$. Define the function $g: X \rightarrow \mathbb{R}$ by $g(x) \stackrel{\text { df }}{=} f\left(x_{0}\right)+\varepsilon$. Let $h=\min \{f, g\}$. Then $h\left(x_{0}\right)=f\left(x_{0}\right)$ and for each neighbourhood $V$ of $x_{0}$ there is $z \in V$ such that $h(z) \in\left(-\infty, h\left(x_{0}\right)-\varepsilon\right] \cup\left\{h\left(x_{0}\right)+\varepsilon\right\}$. By Proposition 2.2, $h \notin \mathcal{U} l s c(X)$

It is easy to see that

Remark 1 Let $X$ be a topological space such that $\mathcal{U}(X)=\mathcal{C}(X)$. Then $\mathcal{M}_{\min }(\mathcal{U} l \operatorname{sc}(X))=\mathcal{C}$.

Now, we recall the definition of a $P$-space [4, pp. 62-63] and two propositions given by Wójtowicz and Sieg [11, Theorem 1 and Corollary 1].

Definition 1 We say that a completely regular (Tychonoff) space $X$ is a $P$-space if every $G_{\delta}$-subset $\left(F_{\sigma}\right.$-subset) of $X$ is open (closed); equivalently, every co-zero subset of $X$ is closed.

Proposition 2.12 Let $X$ be a completely regular space. Then $\mathcal{U}(X)=\mathcal{C}(X)$ if and only if $X$ is a $P$-space.

Proposition 2.13 Let $X$ be a perfectly normal or first countable space, or a locally compact space. Then $\mathcal{U}(X) \neq \mathcal{C}(X)$ if and only if $X$ is non-discrete.

From Proposition 2.12, Theorem 2.11 and Remark 1 we obtain the following Corollary.

Corollary 2.14 Let $X$ be a nonempty completely regular space. Then $\mathcal{M}_{\min }(\mathcal{U}$ lsc $(X))=\emptyset$ if and only if $X$ is not a $P$-space.

Moreover, using Proposition 2.13 and Theorem 2.11 we conclude that

Corollary 2.15 Let X be a non-discrete perfectly normal or first countable space, or a locally compact space. Then $\mathcal{M}_{\min }(\mathcal{U} l s c(X))=\emptyset$.

Finally, observe that we can extend the lists (see e.g. [11, Theorem 1]) of equivalent conditions for $X$ to be a $P$-space as follows:

Corollary 2.16 Let $X$ be a nonempty completely regular space. Then $X$ is a $P$-space if and only if $\mathcal{M}_{\min }(\mathcal{U l s c}(X)) \neq \emptyset$.

\section{Upper semicontinuous functions with a closed graph}

First, we recall some basic property of the functions with a closed graph [3, Proposition 2]

Proposition 3.1 Let $X$ be a topological space. Let $\alpha$ be a real number. If $f \in \mathcal{U}(X)$, then $\alpha \cdot f \in \mathcal{U}(X)$.

From above and the definitions of the classes $l s c(X)$ and $\operatorname{usc}(X)$ we obtain: 
Proposition 3.2 Let $X$ be a topological space. For each function $f \in \mathbb{R}^{X}$ we have $f \in$ $\mathcal{U u s c}(X)$ if and only if $(-f) \in \mathcal{U l s c}(X)$.

Now, we will characterize the following maximal classes for the family of upper semicontinuous functions with a closed graph: the maximal additive class, the maximal multiplicative class and the maximal classes with respect to maximum and minimum.

Theorem 3.3 Let $X$ be a topological space. Then $\mathcal{M}_{a}(\mathcal{U u s c}(X))=\mathcal{U} u s c(X)$.

Proof Observe that, by Proposition 3.2, $f \in \mathcal{M}_{a}(\mathcal{U} \operatorname{usc}(X))$ if and only if $-f \in \mathcal{M}_{a}(\mathcal{U} l s c(X))$. Using Theorem 2.5 and again Proposition 3.2, we conclude that $\mathcal{M}_{a}(\mathcal{U u s c}(X))=\mathcal{U u s c}(X)$.

The next theorem follows from Proposition 3.2.

Theorem 3.4 Let $X$ be a topological space. Then $\mathcal{M}_{m}(\mathcal{U} u s c(X))=\mathcal{M}_{m}(\mathcal{U} l s c(X))$.

Theorem 3.5 Let $X$ be a topological space. Then $\mathcal{M}_{\min }(\mathcal{U} u s c(X))=\mathcal{U} u s c(X)$.

Proof Since $-\min \{f, g\}=\max \{-f,-g\}$ for each functions $f, g \in \mathbb{R}^{X}$, by Proposition 3.2, we conclude that $f \in \mathcal{M}_{\min }(\mathcal{U} \operatorname{usc}(X))$ if and only if $-f \in \mathcal{M}_{\max }(\mathcal{U} l s c(X))$. Now, using Theorem 2.10 and again Proposition 3.2, we obtain that $\mathcal{M}_{\min }(\mathcal{U} u s c(X))=\mathcal{U} u s c(X)$.

It is easy to see that using Theorem 2.11 , Remark 1 and the equivalence $f \in$ $\mathcal{M}_{\max }(\mathcal{U} \operatorname{usc}(X))$ if and only if $-f \in \mathcal{M}_{\min }(\mathcal{U} l s c(X))$, we conclude that:

Theorem 3.6 Let $X$ be a topological space. Then $\mathcal{M}_{\max }(\mathcal{U} u s c(X))=\mathcal{M}_{\min }(\mathcal{U l} \operatorname{sc}(X))$.

Open Access This article is distributed under the terms of the Creative Commons Attribution 4.0 International License (http://creativecommons.org/licenses/by/4.0/), which permits unrestricted use, distribution, and reproduction in any medium, provided you give appropriate credit to the original author(s) and the source, provide a link to the Creative Commons license, and indicate if changes were made.

\section{References}

1. Borsík, J.: Local characterization of functions with closed graphs. Demonstr. Math. 29(3), 643-650(1996)

2. Borsík, J.: Sums, differences, products and quotients of closed graph functions. Tatra Mt. Math. Publ. 24, 117-123 (2002)

3. Doboš, J.: On the set of points o discontinuity for functions with closed graphs. Časopis Pěst. Mat. 110, 60-68 (1985)

4. Gillman, L., Jerison, M.: Rings of continuous functions. Springer, Berlin (1976)

5. Kostyrko, P.: A note on the funtions with closed graphs. Časopis Pěst. Mat. 94, 202-205 (1969)

6. Long, P.E., Hendrix, M.D.: Properties of $c$-continuous functions. Yokohama Math. J. 22, 119-123 (1974)

7. Menkyna, R.: The maximal additive and multiplikative families for functions with closed graph. Acta Math. Univ. Comen. 52(53), 149-153 (1987)

8. Sieg, W.: Maximal classes for the family of quasi-continuous functions with closed graph. Demonstr. Math. 42(1), 41-45 (2009)

9. Szczuka, P.: Maximal classes for lower and upper semicontinuous strong Świątkowski functions. Demonstr. Math. 47(1), 48-55 (2014)

10. Szczuka, P.: The maximal class with respect to maximums for the family of upper semicontinuous strong Świątkowski functions. Math. Slovaca 64(5), 1153-1164 (2014)

11. Wójtowicz, M., Sieg, W.: $P$-spaces and an unconditional closed graph theorem. RACSAM 104, 13-18 (2010) 